\title{
Scanners for Analytic Print Measurement - the devil in the details
}

\author{
Eric K. Zeise, Don Williams, Peter D. Burns and William C. Kress* \\ Eastman Kodak Company, Rochester, NY, USA, 14653-0002 \\ *Toshiba America Business Solutions
}

\begin{abstract}
Inexpensive and easy-to-use linear and area-array scanners have frequently substituted as colorimeters and densitometers for low-frequency (i.e., large area) hard copy image measurement. Increasingly, scanners are also being used for high spatial frequency, image microstructure measurements, which were previously reserved for high performance microdensitometers. In this paper we address characteristics of flatbed reflection scanners in the evaluation of print uniformity, geometric distortion, geometric repeatability and influence of scanner MTF and noise on analytic measurements. Suggestions are made for the specification and evaluation of scanners to be used in print image quality standards that are being developed.
\end{abstract}

Keywords: print quality, image quality, digital scanner

\section{INTRODUCTION}

Over the past few years several image quality standards efforts ${ }^{1,2}$ have addressed printing system performance. The standards have addressed the evaluation of several important attributes, including those for color accuracy, spatial distortion, image sharpness and image macro- and micro-uniformity. Generally the standards have addressed such areas as (digital) test targets, analysis procedures, and print evaluation procedures or measurement devices. In this paper, we address the requirements for print scanners used as part of the evaluations. We will discuss how to interpret the requirements for print quality evaluation in terms of scanner performance.

A goal of the standards development program undertaken by the INCITS W1.1 teams is to specify a set of image quality attributes that correlate with observer-based image quality assessment of hardcopy output devices ${ }^{2}$. A number of attributes have been addressed in the past few years. Here we describe several with an eye to identifying the likely requirements for digital scanners as a measurement device.

\section{UNIFORMITY}

Print macro-uniformity has been defined ${ }^{3}$ as the subjective impression of color across a large area of a single (not printto-print) print sample. The definition of large is $160 \mathrm{~mm}$ by $160 \mathrm{~mm}$. Some macro-uniformity sub-attributes such as mottle, streaks (1-D random line-like structures), bands (1-D uniformly periodic line-like structures), random 2-D noise and the uniformity of color sweeps (vignettes) ${ }^{4}$ are quantifiable using a digital scanner. Attributes requiring absolute rather than relative measurement, such as color reproduction characteristics, are better quantified using a spectrophotometer or colorimeter.

Macro-uniformity measurements require stable low-frequency performance, so MTF is not a primary concern. Tolerances can be translated into scanner requirements by colorimetric calibration. This can be done by transforming tolerances into scanner signal values or transforming scanner signals into colorimetric coordinates. ${ }^{5}$ Typical scannerinduced bias and variation should be kept to some fraction of the test tolerance.

Micro-uniformity assessment examines the deviation from intended uniformity, and includes variations in color. It is differentiated from macro-uniformity by restricting the field of consideration in which the assessment is made. Microuniformity has been defined ${ }^{6}$ as small area (e.g., $6 \mathrm{~mm} \times 6 \mathrm{~mm}$ ) and sub-attributes are streaks, bands, voids (pinhole-type defects), textures (moiré and patterns with correlated phase), satellites and 2-D noise. Many of these can be easily quantified using a capable digital scanner.

The objective of this effort will be to derive micro-uniformity metrics that correlate with observer rankings. Some of the guidelines considered for developing such an objective micro-uniformity metric were: 
1) The metric should be dependent upon the spatial resolution of the human visual system (HVS). By filtering the data using HVS characteristics, only data of visual significance will be considered.

2) There should be an ability to provide a floating reference that accommodates variations in macro-density on a page. That is, there should be no correlation between macro-variations within a page and the micro-uniformity metrics.

3) The spatial characteristics of the image should be preserved and the metrics should be based on these characteristics.

4) The metric must have a quantifiable range that extends from threshold into supra-threshold.

5) It is generally understood that halftoning and noise can mask departures from uniformity and the microuniformity metric must correlate with observer response when these techniques are used,

The procedure of generating these micro-uniformity metrics is being developed, including techniques which (1) digitize uniformity test targets, (2) transform the data into the frequency domain where it is filtered in two dimensions using the HVS spatial resolution characteristic, (3) transform this data back into the spatial domain and (4) calculate the predictors.

Input scanners with a wide range of capabilities are available on the market today and not all are able to produce image quality data that correlate well with human observers. To qualify a scanner for this task, important scanner characteristics need to be assessed such as of bit depth, fixed pattern noise, integrated cavity effects, geometric distortions, dynamic range, scan repeatability, spatial uniformity and the ability to turn off automatic and preprocessing features.

\section{SPATIAL DISTORTION}

The geometric characteristics of a printing process are of particular interest in Graphic Arts applications where image content often crosses page boundaries in a bound pamphlet and precise control of page content is required to avoid creating objectionable artifacts. Typically, the geometric characteristics of a printing process are measured using highend image analyzers employing high magnification video capture integrated with a large area XY positioning table and precision optical encoders. These systems can provide sub-micron precision measurement of geometric features anywhere on an A3/Tabloid sized page. Depending on what image quality attribute is being evaluated, the measurement noise limits vary from a fraction of a micron for particle shape and some edge stairstep measurements to about ten microns for some image content placement measurements. Large area reflection scanners available for a small fraction of the cost of a full motion integrated image analysis system appear to have the capability to perform some of these measurements. The motion control systems of reflection scanners control the positioning of the scan elements to a fairly high accuracy, but some compromises are necessary to maintain a competitive cost. The different optical systems employed by scanners introduce their own features. For quantitative analysis of geometric image quality attributes, the important characteristics of a scanner describe how well the scanner can reproduce the geometric characteristics of the original print. Among the geometric characteristics of a reflection scanner, which may be different in the fast scan and slow scan directions, are starting position jitter of the scan, scale factor, geometric distortion and geometric accuracy.

For multiple scans of the same print, without moving the print on the scanner platen, there is some jitter in the actual starting position of the scan. This starting position jitter is illustrated in Fig. 1 for a fairly high quality A3/Tabloid size reflection scanner. The jitter distribution is arguably a normal distribution. If so, the standard deviation of this distribution, about 33 microns, represents an error in where the scan starts that is a significant fraction of the registration budget for a high quality printer. An overscan of the print against a complementary background (e.g. black background for white paper) provides paper edge information that allows an analysis algorithm to compensate for starting position jitter. 


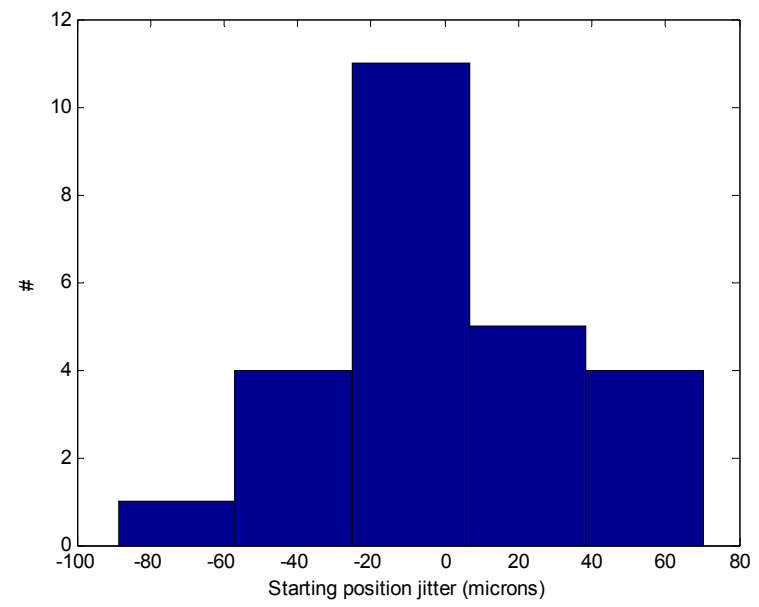

Figure 1: Scanner starting position jitter

A useful target for evaluating magnification, geometric distortion, and geometric accuracy is a grid target. High precision, NIST traceable dot grid patterns are available from several vendors. We evaluated the optical resolution of our A3/Tabloid scanner with a metal on ceramic dot grid target. The optical resolution of this scanner, the highest resolution for which no pixel interpolation should be occurring, is specified as 1600 dpi. No pixel interpolation should occur for integer fractions of this optical resolution. At an 800 dpi scanner driver setting, with the quiescent scanner at 20 degrees Centigrade, the measured slow scan scale factor was $801.3 \mathrm{dpi}$, the fast scan scale factor was $800.3 \mathrm{dpi}$. Overall, this is a less than $0.2 \%$ magnification error, but for a scanner employed in geometric measurement such a magnification error can result in a measured positional error of over half a millimeter across the entire A3/Tabloid scanner platen area if the magnification error is not properly calibrated and compensated.

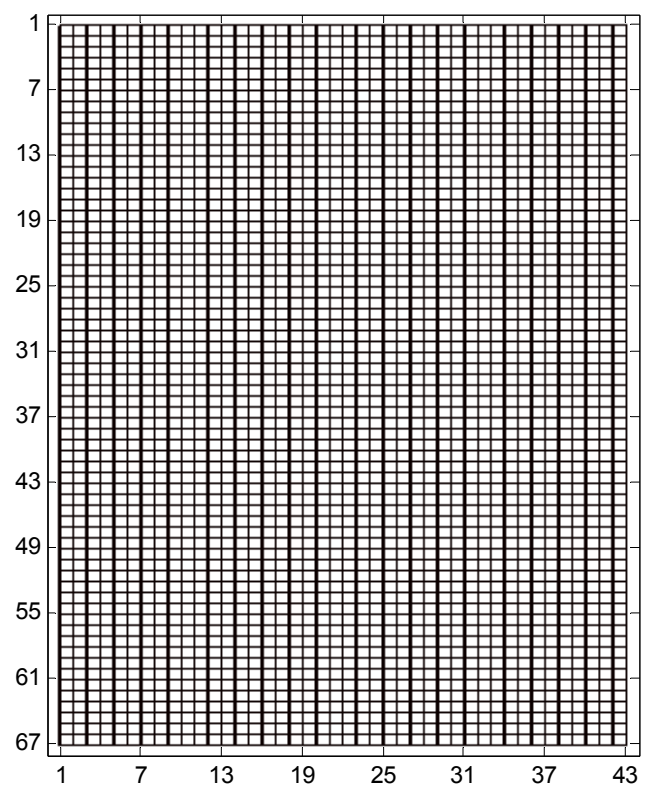

Figure 2: Line grid target for scanner characterization 
As the materials used in constructing a scanner expand and contract with temperature, the magnification of a scanner will be slightly temperature dependent. For a 10 degree Centigrade increase in temperature the platen of a Tabloid sized scanner will expand by almost 40 microns.

Metal on ceramic dot grid targets are not easily available in tabloid size, so we have taken a slightly different approach by printing a line grid target consisting of 43 vertical and 67 horizontal lines at 0.25 inch intervals over the area of a tabloid sized sheet and then calibrating the grid positions using a high magnification image analyzer equipped with a large X-Y table and high-precision optical encoders ${ }^{7}$. Figure 2 illustrates this $11 \times 17$ inch target.

Scans of this calibrated line grid target can then be used to evaluate the geometric distortion and accuracy characteristics of a scanner. The process used was to measure the horizontal and vertical grid line trajectories in the scanned bitmap of the line grid target. The difference between the grid positions measured from the scanned bitmap of the target and the grid positions of the target measured using the high precision video analysis system provides a measure of the geometric accuracy of the scanner over the entire $11 \times 17$ inch area of the scan. These differences, evaluated for example at the intersection points of the grid lines, can be used to create a map of error vectors or a false color map of error magnitude over the scanner imaging area. Through examination of the trends in these error maps, and the stability of the error maps in repeated scans, one can determine whether the geometric distortion of the scanner is sufficiently stable to enable calibration and compensation thus providing a fast and fairly accurate measure of the geometric position of features in a large area scanned target.

\section{SCANNER MTF}

In addressing the requirements for the capture of fine spatial detail in scanned test prints, it is natural to consider adopting standard methods by which digital scanners and cameras are evaluated. The well-established standard, ISO 12233 method $^{8,9}$ of the spatial frequency response (SFR), based on the analysis of slanted-edge features has been applied and adopted for print scanner evaluation in ISO 16067-1. This method, based on edge-gradient analysis, results in a function of spatial frequency. When corrected for the input (target) edge modulation, this measurement method results in an effective MTF.

Standard methods for several print quality attributes can be interpreted in terms of scanner MTF requirements. For example, Fig. 3 shows the frequency weighting used in text quality evaluations, reported by Wu and Dalal ${ }^{10}$. In order to apply such a sensitivity weighting to image structure signals, a relatively high and stable MTF over this range is needed. To cover such a bandwidth, $[0-8 \mathrm{cy} / \mathrm{mm}$ ], one could conclude that $400 \mathrm{ppi}$ sampling would be sufficient. Usually, however, significantly higher scanner sampling is needed, to limit aliasing artifacts from high-frequency image fluctuations due to, e.g., digital halftoning.

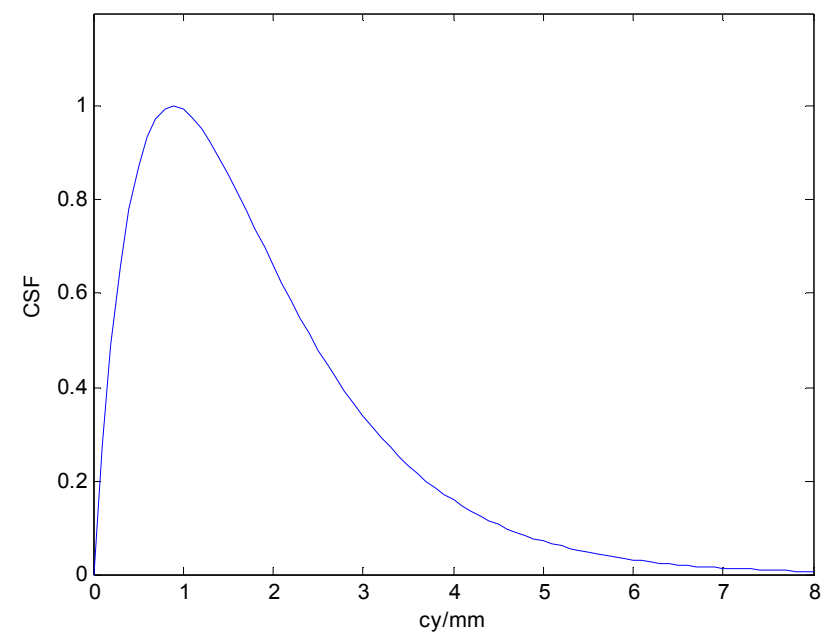

Figure 3: Spatial frequency weighting used in text quality metrics 
Scanner requirements can also be interpreted for line-raggedness measurements. Fig. 4 from Ref. 11 shows an example based on an inkjet print measurement. Here the Tangential Edge Profile (TEP) is found using slanted-edge analysis, and the fluctuations from the nominal edge location are expressed as a TEP noise-power spectrum. In this example the frequency weighting was somewhat different from that of Fig. 3, but similar in bandwidth.

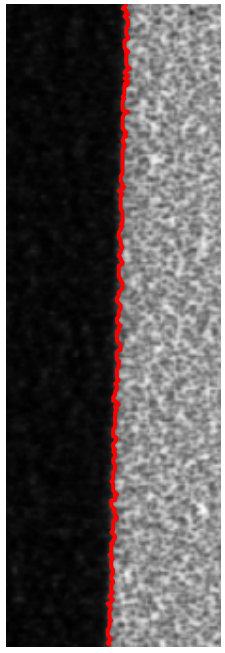

(a)

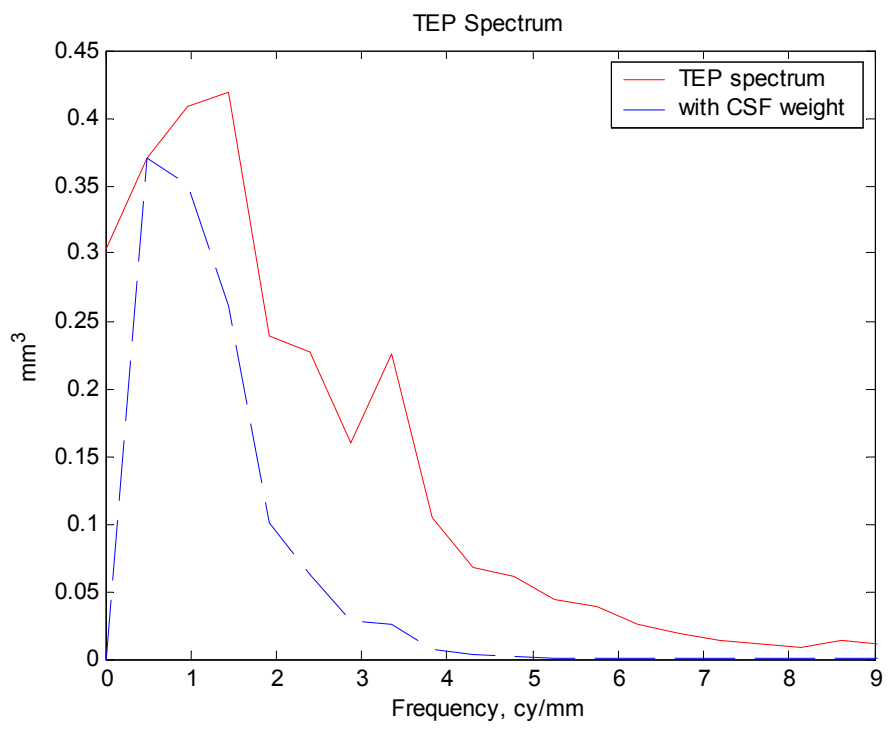

(b)

Figure 4: (a) Scanned inkjet print with detected edge added, and (b) TEP noise-power spectrum and CSF weighting for $33 \mathrm{~cm}$ viewing distance, from ref. 11

\section{IMAGE NOISE}

When selecting a digital scanner for print evaluation it is useful to understand the magnitude and nature of unwanted image fluctuations, often referred to as noise. For several sources of this image noise, a statistical description is appropriate ${ }^{12,13}$. Other sources have both stochastic and predictable components. Pixel-to-pixel detector sensitivity variations can introduce repeatable patterns into the image data and may appear to be random from unit to unit, but somewhat repeatable from scan-to-scan for a given scanner. An additional source of apparent noise is the test target used in the measurement. This can be due to, e.g., surface texture, scratches, or the microstructure of the printing technology used. It is usually helpful to specify the acceptable levels for the repeatable- (fixed-pattern), and random, (temporal) noise components. Ref. 14 describes methods to do this.

Just as the signal-transfer characteristics of digital scanners can be expressed as a function of spatial frequency (MTF), so to can the random noise introduced. Several print-quality attributes, such as banding and graininess are evaluated in this way ${ }^{15}$. Figure 5, from Ref. 16 shows the apparent image noise introduced by a desktop scanner used for print graininess evaluation. The top plot (a) shows the observed image noise from a scan of a low-noise photographic test target. The lower plot (b) presents the corresponding spectrum from a scan of an inkjet print of the same optical density. By comparing the scanned print (scanner plus print) noise levels of fig. 5(b), with those attributable to scanner alone, practical scanner performance criteria can be established. 


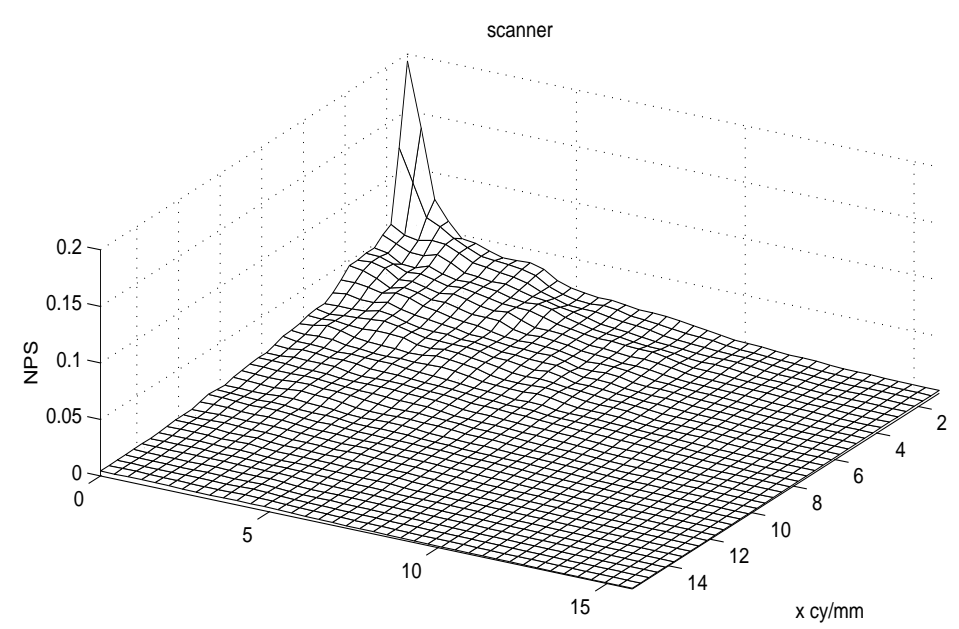

(a)

$\mathrm{vcv} / \mathrm{mm}$

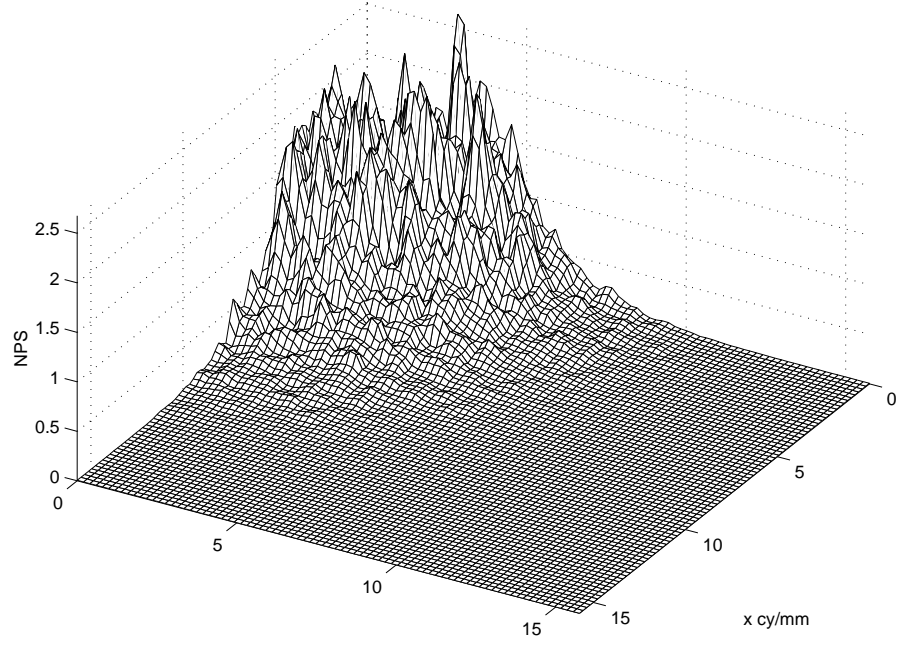

(b)

$\mathrm{y} \mathrm{cy/mm}$

Figure 5. (a) Example scanner-and, (b) printer noise-power spectra

\section{CONCLUSIONS}

When selecting a digital scanner for print image quality measurement, it is helpful to translate the requirements into corresponding standard performance measures developed for digital camera and scanner evaluation. The capture of image detail for text quality and micro-uniformity set requirements for scanner MTF and image noise. For noise fluctuations introduced by the scanner, attention should be paid to the magnitude and spatial-frequency components of any fixed-pattern and random-temporal sources. Requirements for low levels of spatial distortion, however, often call for tolerance levels similar to those for graphics-arts scanning, rather than lower-cost desktop models. In addition to the above imaging performance, a level of control over the operating parameter and image processing functions is also required.

\section{REFERENCES}

1. ISO/IEC 13660:2001 Information Technology - Office Equipment - Measurement of image quality attributes for hardcopy output - Binary monochrome text and graphics images, ISO, 2001.

2. INCITS W1.1, Appearance-based image quality standards for printers, http://www.incits.org/tc home/w11/incits w11.htm 
3. D. R. Rasmussen, K. D. Donohue, Y. S. Ng, William C. Kress, F. Gaykema, S. Zoltner ISO 19751 macro-uniformity, Proc. SPIE-IS\&T Electronic Imaging Symposium, SPIE vol. 6059, pp. 200-206, 2006

4. S. Farnand, F. Gaykema, W. Kress, O. Martinez, A. McCarthy, H.n Shin, E. Zeise, Update on the INCITS W1.1 Standard for Evaluating the Colour Rendition of Printing Systems, Proc. SPIE-IS\&T Electronic Imaging Symposium, SPIE vol. 6059,2006

5. W. C. Kress, Digitization and Metric Conversion for Image Quality Test Targets, Proc. IS\&T PICS Conf., pp. 82-87, 2003.

6. B. Mishra, D. R. Rasmussen, Micro-uniformity: An Image Quality Metric for Measuring Noise, Proc. IS\&T PICS Conf, pp. 108-113, 2000.

7. ImagExpert Inc., 486 Nashua Street, Nashua, NH 03063 USA. (www.imagexpert.com)

8. ISO 12233, Photography - Electronic still picture cameras - Resolution measurements, ISO, 1998.

9. S. E. Reichenbach, S. K. Park, and R. Narayanswamy, Characterizing Digital Image Acquisition Devices, Opt. Eng., 30, 170177,1991

10. W. Wu and E. N. Dalal, Perception-based Line Quality Measurement, Proc. SPIE-IS\&T Electronic Imaging Symposium, SPIE vol. 5668, 2005

11. P. D. Burns, Edge-raggedness Evaluation Using Slanted-edge Analysis, Proc. SPIE-IS\&T Electronic Imaging Symposium, SPIE vol. 6059, 200-206, 2006.

12. P. D. Burns, Image Signal Modulation and Noise Characteristics of Charge-Coupled Device Imagers, Proc. SPIE, 1071, pp. 144$152,1989$.

13. C. S. McCurnin, L. C. Schooley, and G. R. Sims, Charge-Coupled Device Signal Processing Models and Comparisons, J. Electronic Imaging, 2, pp. 100-107, 1993.

14. P. D. Burns and D. Williams, Identification of image noise sources in digital scanner evaluation, Proc. SPIE-IS\&T Electronic Imaging Symposium, SPIE vol. 5294, 114-123, 2004.

15. P. J. Kane, T. F. Bouk, P. D. Burns, and A. D. Thompson, Quantification of Banding, Streaking, and Grain in Flat Field Images, Proc. PICS, pp. 79-83, Portland, OR, 2000.

16. J. C. Stanek and P. D. Burns, Scanning Parameter Selection for Inkjet Print Analysis, Proc. PICS Conf., 135-139, IS\&T, 2003.

Proc. SPIE Vol. 6494, Image Quality and System Performance IV, 64940L, 2007 